http://dx.doi.org/10.32929/2446-8355.2019v28n1p97-110

\title{
ESTIMATIVA DA CURVA DE RETENÇÃO DE ÁGUA NO SOLO POR FUNÇÃO DE PEDOTRANSFERÊNCIA
}

\author{
Jhosefe Bruning ${ }^{1 *}$, Adroaldo Dias Robaina ${ }^{2}$, Marcia Xavier Peiter ${ }^{2}$, Ricardo Boscaini ${ }^{3}$, \\ Chaiane Guerra da Conceição ${ }^{1}$, Elisa de Almeida Gollo ${ }^{3}$
}

\footnotetext{
${ }^{1}$ Engenheiro Agrícola, M.Sc., Doutorando(a) do Programa de Pós-Graduação em Engenharia Agrícola, da Universidade Federal de Santa Maria (UFSM), Campus Santa Maria - Departamento de Engenharia Rural, Centro de Ciências Rurais. Prédio 42, $3^{\circ}$ Piso, CEP 97105-900, Santa Maria - RS. * E-mail do autor correspondente: jhosefe.b@gmail.com.

${ }^{2}$ Engenheiro(a) Agrônomo(a), Dr.(a), Professor(a) do Centro de Ciências Rurais, Departamento de Engenharia Rural, Universidade Federal de Santa Maria (UFSM), Santa Maria - RS, Brasil.

${ }^{3}$ Engenheiro(a) Agrônomo(a), M.Sc., Doutorando(a) do Programa de Pós-Graduação em Engenharia Agrícola, Universidade Federal de Santa Maria (UFSM), Santa Maria - RS, Brasil.
}

Recebido: 23/01/2018; Aceito: 04/06/2019

RESUMO: A curva característica de água no solo é um importante fator para descrever o comportamento físico-hídrico dos solos pois é de grande importância na agricultura, por apresentar aplicações científicas, técnicas e práticas. Desta forma, a estimativa da mesma pode ser feita através de diversos métodos, sendo o mais utilizado o método da câmara de Richards. Assim, o objetivo desse trabalho foi avaliar o modelo proposto por Arya e Paris, para estimar a umidade volumétrica para duas classes de solo, com base na análise textural. Foram selecionados na literatura solos oriundos de duas classes texturais e três locais de origem, onde foram utilizados os dados texturais para realizar a estimativa da curva de retenção de água no solo através dos parâmetros de Van Genuchten gerados através do programa Qualisolo e comparar com a curva gerada pelo método direto. Os resultados obtidos mostraram uma subestimação dos valores de umidades estimadas para os solos analisados, apresentaram coeficiente de determinação superiores a 0,87 relatando uma boa correlação entre as umidades observadas e estimadas. Portanto, conclui-se que os resultados mostraram uma boa alternativa de uso do método proposto por Arya e Paris para gerar a curva de retenção de água no solo de maneira satisfatória.

Palavras-chave: Arya e Paris. Van Genuchten. Umidade volumétrica. Textura do solo.

\section{ESTIMATION OF SOIL WATER RETENTION CURVE BY PEDOTRANSFERENCE FUNCTION}

ABSTRACT: The soil water characteristic curve is an important factor for describing the physical and water behavior of soils, since it is of great importance in agriculture because it presents scientific, technical and practical applications. In this way, the estimation can be done through several Methods, being the most used the method of the camera of Richards. Thus, the objective of this work was to evaluate the model proposed by Arya and Paris, to estimate the volumetric moisture for two soil classes, based on the textural analysis. Soils 
from two textural classes and three sites of origin were selected in the literature, where textural data were used to estimate the soil water retention curve by the Van Genuchten parameters generated by the Qualisolo program and compare with the curve generated by the direct method. The results showed an underestimation of the estimated values of the soils analyzed, presented coefficient of determination superior to 0,87 , reporting a good correlation between the observed and estimated humidities. Therefore, it was concluded that the results showed that is a good alternative use the method proposed by Arya and Paris to satisfactory generate the soil water retention curve.

Key words: Arya and Paris. Van genuchten. Volumetric moisture. Soil texture.

\section{INTRODUÇÃO}

A curva característica de água no solo é importante na descrição do comportamento físico-hídrico dos solos não saturados (LUCAS et al., 2011). No entanto, de difícil caracterização, principalmente pelo tempo consumido nos procedimentos laboratoriais para a obtenção dos parâmetros da curva de retenção de água (CRA). Tem grande importância na agricultura, pois apresenta a determinação dos limites de saturação, umidade residual e da disponibilidade total de água no solo (NASCIMENTO, 2009).

Além disso, a curva de retenção de água no solo é de suma importância para o manejo da irrigação onde, através de seus parâmetros, é possível determinar quando e quanto irrigar, essencial também para estabelecer condições de manejo adequadas para a proteção ambiental em sistemas agrícolas e sendo ainda caracterizado como uma forma de medida indireta de distribuição do tamanho dos poros do solo (REINERT; REICHERT, 2006; SCORZA JÚNIOR et al., 2010).

Dentre as metodologias empregadas para a obtenção da CRA do solo, destaca-se o método direto descrito por Richards (1965). Limitações inerentes aos métodos convencionais são a elevada ocupação de espaço físico pelos equipamentos, forte dependência de pessoas habilitadas a operar os equipamentos e o elevado prazo de resposta dos laboratórios para a determinação da curva de retenção (VAZ et al., 1999; NAIME et al., 2001; ANTINORO et al., 2014; SILVA; ARMINDO, 2016).

Neste sentido, é crescente a utilização de funções de pedotransferência que utilizam a análise textural para estimativa dos parâmetros empíricos dos modelos. Dentre os modelos destaca-se o de Van Genuchten (1980), considerado o modelo mais utilizado, por apresentar bom ajuste à curva experimental para uma grande variedade de solos (XIANG-WEI et al., 2010). As funções de pedotransferência permitem a previsão dos parâmetros hidráulicos de van Genuchten e da condutividade hidráulica saturada através da utilização de dados texturais dos solos como entrada, tornando assim, uma ferramenta útil para os locais com limitações de dados.

Em estudo realizado por Melo et al., (2015), utilizando funções de pedotransferência para predizer o comportamento da curva de retenção de água para solos brasileiros, foram encontrados resultados satisfatórios em relação aos métodos testados para os solos analisados. 
Dentre os procedimentos disponíveis que utilizam como base análise textural, destacase o método desenvolvido por Arya e Paris (1981), que utiliza a distribuição de partículas por tamanho para obter a CRA no solo. Além disso, a utilização do programa Qualisolo tem como função aplicar o método proposto pois, apresenta elevado potencial de utilização para os solos do Brasil, tendo a possibilidade de estimar as curvas de retenção através de dados de granulometria, densidade do solo e densidade das partículas dos solos, disponíveis na maioria dos estudos de levantamento e manejo de solos (VAZ et al., 2007).

Diante do exposto, este trabalho objetivou avaliar o desempenho do procedimento proposto por Arya e Paris, para estimar os parâmetros do modelo de Van Genuchten em três solos característicos de áreas irrigadas, utilizando como base de entrada a análise textural e comparando com a umidade volumétrica observada.

\section{MATERIAL E MÉTODOS}

O presente estudo foi realizado com dois tipos de solo oriundos de três locais distintos, sendo classificados como Argissolo Vermelho Distrófico arênico (EMBRAPA, 2005) localizados nos municípios de Alegrete com latitude Sul 29 $42^{\prime}$ 52,85" e longitude Oeste $55^{\circ}$ 31' 29,69", Argissolo Vermelho Distrófico arênico localizado no município de Santa Maria situada na latitude Sul 29॰41'2 4" e longitude Oeste 5348'42" e Latossolo Vermelho distrófico típico localizado no município de Santiago latitude Sul 29० 09' 50" e Oeste $54^{\circ} 51^{\prime}$ " 32" ambos os solos classificados segundo (EMBRAPA, 1999), as três áreas estão localizadas no estado do Rio Grande do Sul (RS). As coletadas de solos para os três municípios foram realizadas por meio de amostras ao longo do perfil do solo. Os solos utilizados são característicos de regiões produtoras de grandes culturas, tais como arroz, soja e milho.

Foram utilizados os seguintes dados texturais: Areia, Silte e Argila (\%), densidade de partículas (dp), densidade aparente do solo (ds) e umidade volumétrica, medidos em diferentes tensões $(0,001,0,006,0,01,0,033,0,1,0,5,1,5 \mathrm{MPa})$, sendo estes dados obtidos de Torres (2014), Oliveira (2011) e Gomes (2011), mostrados na Tabela 1. Através dos dados de granulometria (\%), densidade do solo (g cm-3) e de densidade de partículas ( $\mathrm{g} \mathrm{cm}-3)$, foi feita a obtenção dos parâmetros de ajuste $(\theta \mathrm{r}, \theta \mathrm{s}, \mathrm{n}, \mathrm{m}$ e $\alpha)$ pelo software Qualisolo 2009. Para realizar a estimativa da curva de retenção de água no solo utilizou-se o modelo de Van Genuchten (1980). 
Tabela 1. Valores de areia, silte e argila (\%), densidade do solo e densidade de partículas (g $\mathrm{cm}^{-3}$ ) para solos oriundos de três locais. Sand, silt and clay values (\%), soil density and particle density $\left(\mathrm{g} \mathrm{cm}^{-3}\right)$ for soils from three locations.

\begin{tabular}{cccccc}
\hline \multirow{2}{*}{ Profundidade $(\mathrm{cm})$} & \multicolumn{3}{c}{ Granulometria $(\%)$} & \multicolumn{2}{c}{ Densidade $\left(\mathrm{g} \mathrm{cm}^{-3}\right)$} \\
\cline { 2 - 5 } & Areia & Silte & Argila & Solo & Partícula \\
\hline \multicolumn{5}{c}{ Argissolo Vermelho distrófico arênico (1) * } \\
\hline $5-5$ & 69,61 & 12,51 & 17,88 & 1,77 & 2,6 \\
$5-15$ & 69,03 & 11,72 & 19,24 & 1,69 & 2,58 \\
$15-30$ & 63,9 & 12,73 & 23,37 & 1,67 & 2,62 \\
$30-45$ & 60,02 & 12,1 & 27,89 & 1,64 & 2,58 \\
$45-60$ & 56,85 & 12,29 & 30,86 & 1,66 & 2,6 \\
$60-75$ & 56,2 & 11,66 & 32,14 & 1,66 & 2,65 \\
\hline \multicolumn{5}{c}{ Argissolo Vermelho distrófico arênico (2) ** } \\
\hline $0-10$ & 36 & 44,7 & 19,4 & 1,39 \\
$10-25$ & 35,5 & 40,4 & 24,2 & 1,35 & 2,59 \\
$25-55$ & 32,1 & 35,4 & 32,5 & 1,42 & 2,63 \\
$55-85$ & 24,2 & 31,7 & 44,1 & 1,59 & 2,67 \\
\hline $0-20$ & Latossolo Vermelho distrófico típico (3) *** & \\
\hline $20-40$ & 49,8 & 25,63 & 24,6 & 1,55 & 2,6 \\
$40-60$ & 45,77 & 24,47 & 29,77 & 1,39 & 2,6 \\
$60-80$ & 40,37 & 21,47 & 34,77 & 1,34 & 2,63 \\
$80-100$ & 37,97 & 22,27 & 39,77 & 1,35 & 2,62 \\
\hline
\end{tabular}

*Argissolo Vermelho distròfico arênico (1) referente ao município de Alegrete, **Argissolo Vermelho distròfico arênico (2) referente ao município de Santa Maria, ***Latossolo Vermelho distrófico típico (3) referente ao município de Santiago. *Typic Paleudult (1) of the city of Alegrete, **Typic Paleudult (2) of the city of Santa Maria, *** Typic Hapludox (3) of the city of Santiago.

Com base nos parâmetros de ajuste obtidos e utilizando a equação de Van Genuchten, realizou-se a determinação das umidades estimadas de todos os pontos e tensões analisadas através da equação (1).

$$
\theta=\theta \mathrm{r}+\frac{\theta \mathrm{s}-\theta \mathrm{r}}{\left[1+\left(\alpha|\psi \mathrm{m}|^{n}\right)\right]^{n}}
$$

onde: $\theta$ : Umidade volumétrica do solo; $\theta \mathrm{r}$ e $\theta$ s: Umidade residual e de saturação do solo, respectivamente; $\Psi \mathrm{m}$ : Potencial matricial da água no solo; $\alpha, \mathrm{n}$ e m: Parâmetros de ajuste do modelo de Van Genuchten, que correspondem a forma e inclinação da curva.

De posse dos dados de umidade volumétrica observados, através dos métodos diretos e utilizando os dados de umidade estimados através dos parâmetros obtidos pelo software (método indireto) foram feitas as comparações. Utilizaram-se as seguintes análises: raiz do quadrado médio do erro (RQME), o erro absoluto médio (EAM) e índices demonstrativos de desempenho estatístico. Este desempenho foi avaliado pelo coeficiente de determinação $\left(\mathrm{R}^{2}\right)$, índice de correlação (r) e índice de exatidão (d) (WILLMOTT et al., 1985). Conforme 
apresentado na equação (2) o índice d varia de 0 para nenhuma concordância, a 1 para concordância perfeita.

$$
d=1-\left[\frac{\sum_{i=1}^{n}\left(y_{i}-x_{i}\right)^{2}}{\sum_{i=1}^{n}\left(\left|y_{i}-0\right|+\left|x_{i}-0\right|\right)^{2}}\right]
$$

onde: d: Índice de concordância de Willmott; yi: Teor de água no solo estimado pelo modelo; x i: Teor de água no solo observado; o: Média dos valores observados.

Com o intuito de dar suporte à análise, determinou-se o índice de desempenho ou concordância (c) através da equação (3), assim os resultados foram interpretados conforme descrição da Tabela 2, sugerida por Camargo e Sentelhas (1997).

$$
c=r x d
$$

onde: c: Índice de desempenho; r: Índice de correlação.

Tabela 2. Critérios para a classificação do desempenho através do índice c. Criteria for the classification of performance by the $c$ index.

\begin{tabular}{cc}
\hline Valores de "c" & Desempenho \\
\hline$>0,85$ & Ótimo \\
0,76 a 0,85 & Muito Bom \\
0,66 a 0,76 & Bom \\
0,61 a 0,65 & Mediano \\
0,51 a 0,60 & Sofrível \\
0,41 a 0,50 & Mau \\
$\leq 0,41$ & Péssimo \\
\hline
\end{tabular}

Fonte: Camargo e Sentelhas,1997.

\section{RESULTADOS E DISCUSSÃO}

Nas Figuras 1, 2 e 3 são apresentados os valores de umidade volumétrica em função da tensão de água no solo para as diferentes profundidades e classe de solos. Com base nos dados analisados foi possível observar similaridade entre os resultados obtidos para as diferentes profundidades e tensões avaliadas, corroborando com resultados encontrados por Nascimento et al. (2010) onde verificaram uma similaridade em relação a quantidade de água retida no solo, em função das tensões aplicadas nas diferentes profundidades estudadas.

$\mathrm{Na}$ comparação entre as curvas, para as diferentes profundidades dos solos analisados, observou-se uma tendência de maior proximidade e uniformidade entre valores observados e estimados para as tensões superiores a 0,1 MPa (Figura 1, 2 e 3). Já para tensões inferiores a 0,1 MPa foi possível observar maior discrepância entre os valores de umidade observados com os dados estimados, concordando com Nascimento et al., (2010), onde encontraram um comportamento semelhante quando comparado com os demais métodos estudados. De modo geral, os resultados encontrados apresentaram a tendência de aproximação com os valores observados para as tensões mais próximas de 1,5 MPa do que para as tensões menores, subestimando os valores observados. Estes resultados são similares aos encontrados por Nebel et al. (2010) e Khodaverdiloo et al. (2011) que relataram a subestimação dos valores de 
umidade para as tensões maiores. Outros autores como Rezaee et al. (2011) e Melo et al., 2015 também identificaram em análise feita no programa Qualisolo, que o valores de umidade foram subestimados tanto para valores altos como para valores baixos do potencial matricial.

A concordância entre os valores estimados e observados em menores tensões pode ser pelo fato de a retenção de água no solo estar mais associado ao arranjo das partículas do solo do que com as forças de adsorção das partículas, conforme citado por Campelo Junior et al., (2014). Esses autores salientam que a textura é uma característica empregada como base para a função de pedotransferência utilizada neste estudo. Os comportamentos das curvas de retenção de água no solo nos três locais apresentaram uma tendência de subestimação das umidades observadas nas mesmas tensões aplicadas.

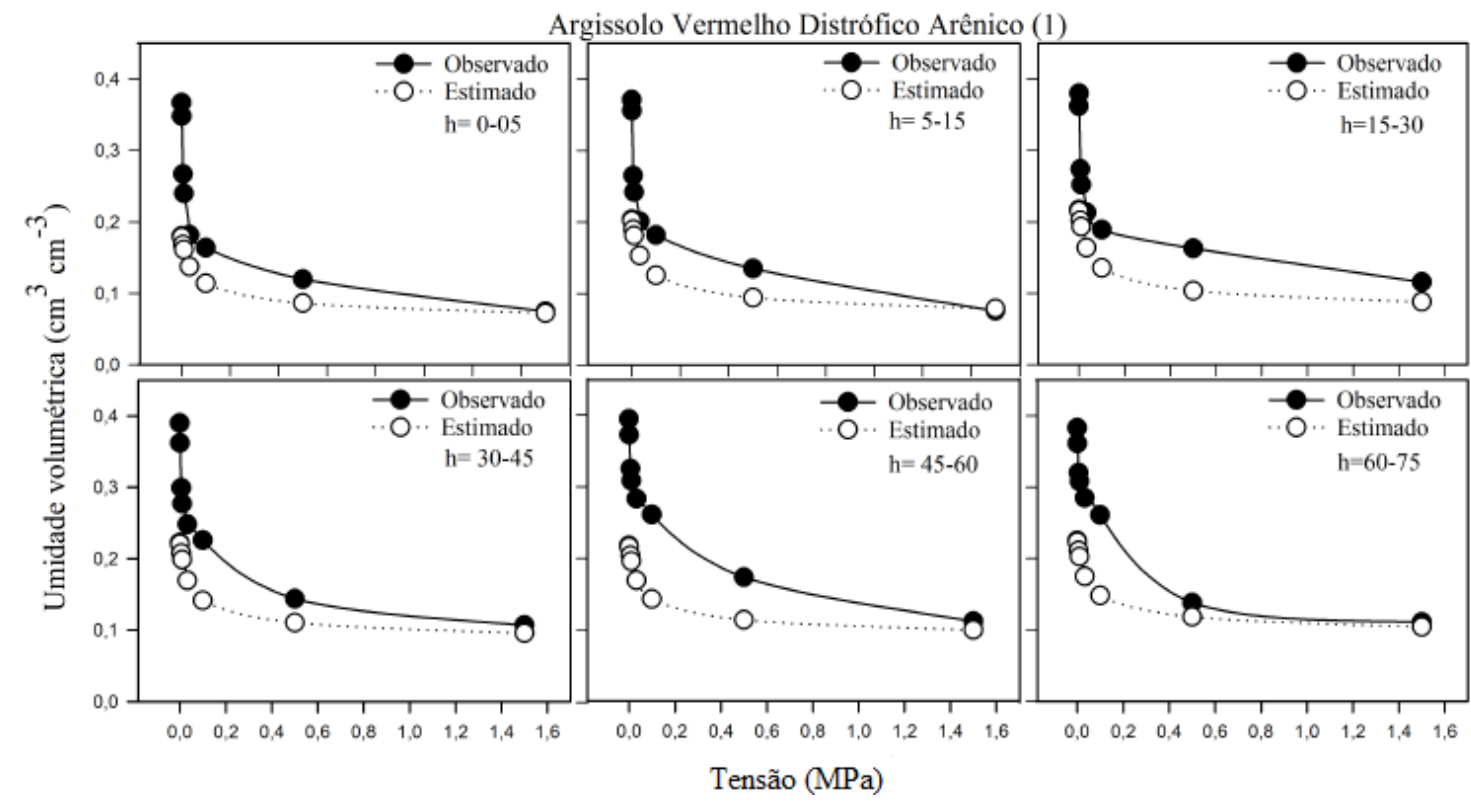

Figura 1. Comportamento da umidade volumétrica em função da tensão para o Argissolo Vermelho distrófico arênico (1) nas diferentes profundidades analisadas. Volumetric moisture as a function of the tension for the Typic Paleudult (1) at different depths. 


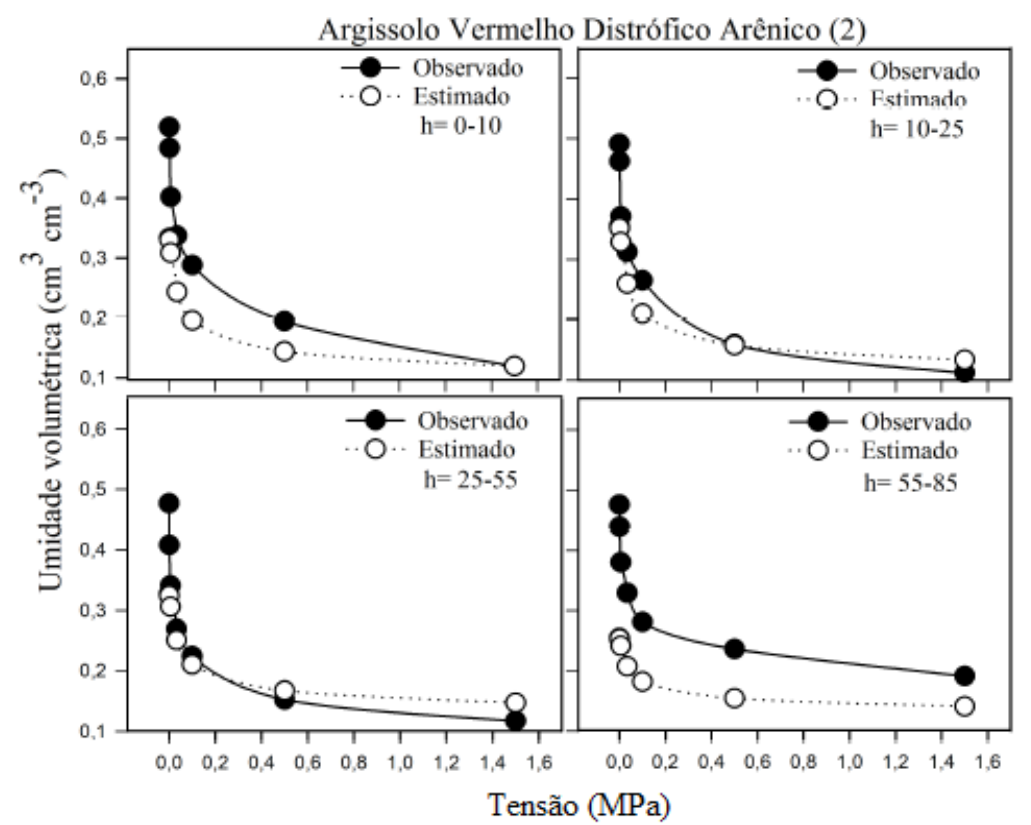

Figura 2. Comportamento da umidade volumétrica em função da tensão para o Argissolo Vermelho distrófico arênico (2) nas diferentes profundidades analisadas. Volumetric moisture as a function of the tension for the Typic Paleudult (2) at different depths.

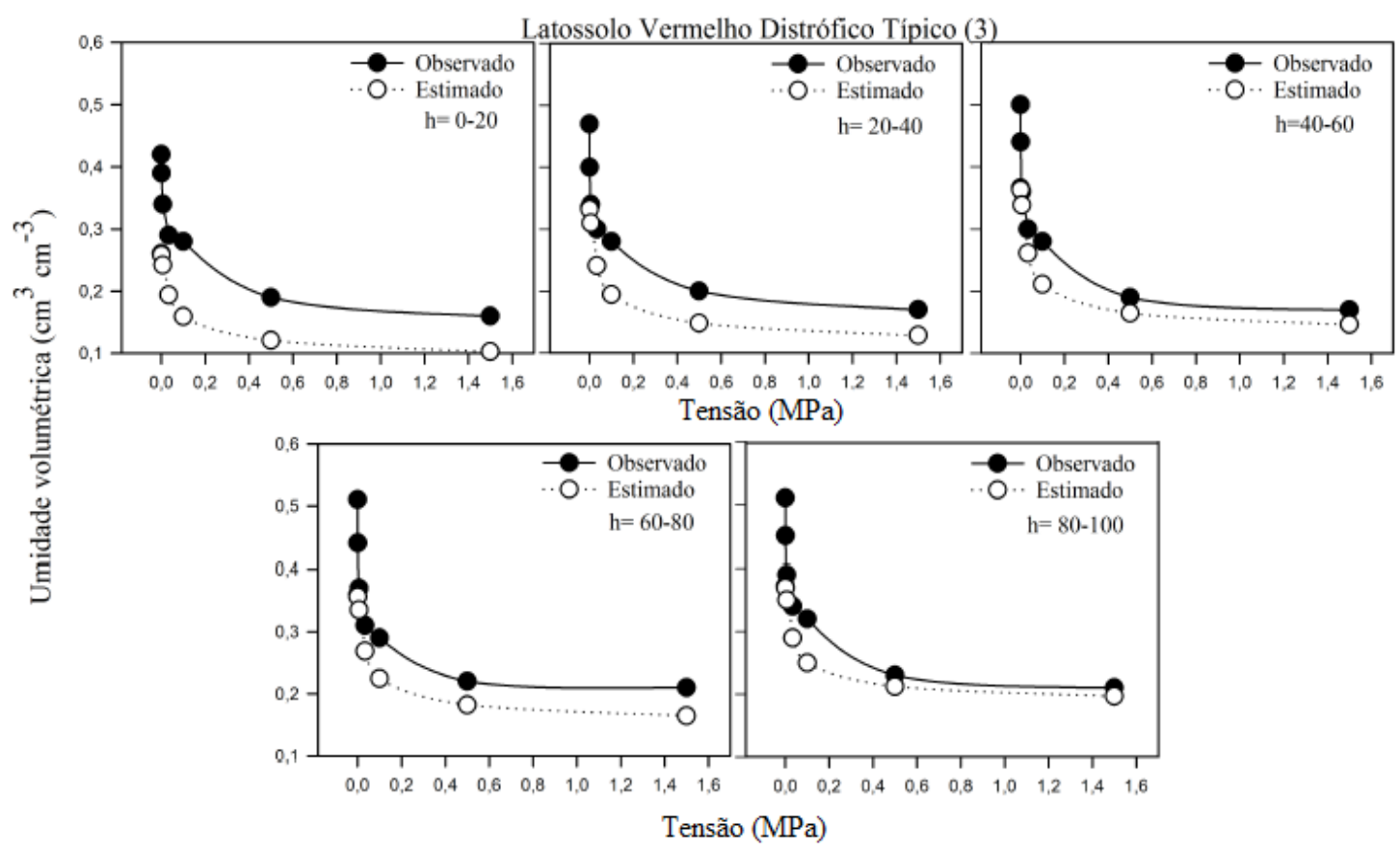

Figura 3. Comportamento da umidade volumétrica em função da tensão para o Latossolo Vermelho distrófico típico (3) nas diferentes profundidades analisadas. Volumetric moisture as a function of the tension for the Typic Hapludox (3) at different depths.

Nas Figuras 4, 5 e 6 são apresentados os dados de umidade volumétrica observados em função dos dados estimados para as diferentes profundidades e classes texturais. Com base nas análises realizadas pode ser verificado que todas as profundidades, apresentaram R2 superiores a 0,87 relatando uma boa correlação entre as umidades observadas e estimadas, concordando com Barros et al., (2013), que utilizaram diferentes funções de pedotransferência 
(PTFs) para estimar o conteúdo de água através de dados de textura do solo, para o Estado de Santa Catarina.

A partir dos dados analisados, pode-se destacar que o Argissolo Vermelho distrófico arênico (2) de Santa Maria e o Latossolo Vermelho distrófico típico (3) de Santiago, apresentaram os melhores R2, 0,97 e 0,96, respectivamente.

Porém a simples adoção do $\mathrm{R}^{2}$ não pode ser o único critério levado em consideração para definir a qualidade de métodos, pois o mesmo não estabelece o tipo e a magnitude das diferenças entre um valor padrão e um valor estimado (BARROS et al., 2009). Sendo assim, a análise dos índices de concordância de Wilmontt (d), a raiz do quadrado médio do erro (RQME) e o erro absoluto médio (EAM) auxiliam a interpretação dos resultados encontrados.

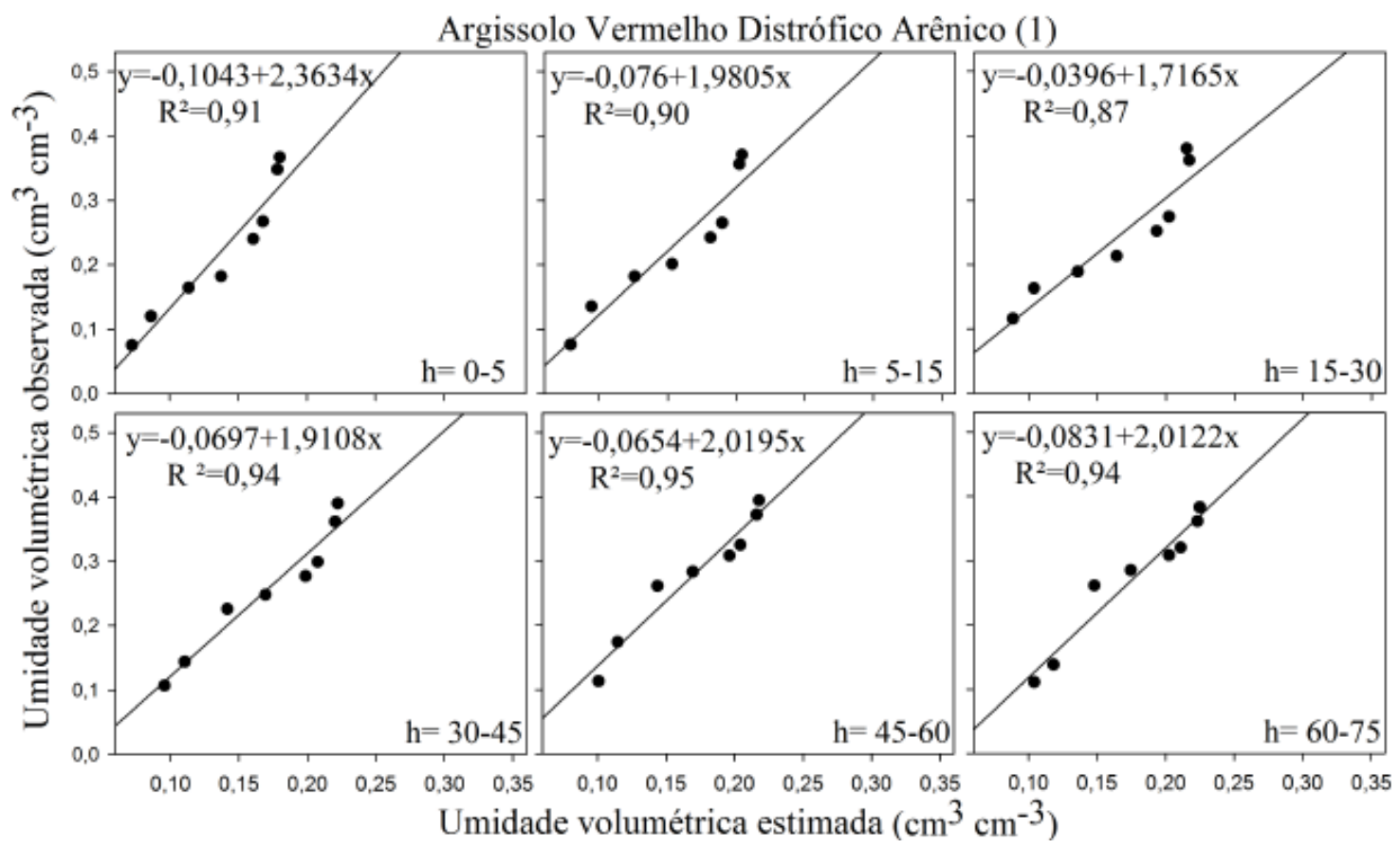

Figura 4. Representação gráfica da estimativa da umidade volumétrica obtida através do método direto, comparada com os valores de umidades obtidos através dos parâmetros gerados pelo Qualisolo para o Argissolo Vermelho distrófico arênico (1) nas diferentes profundidades analisadas. Graphical representation of the volumetric moisture estimation obtained by the direct method, compared to the values obtained by the parameters generated by the Qualisolo for the Typic Paleudult (1) at different depths. 


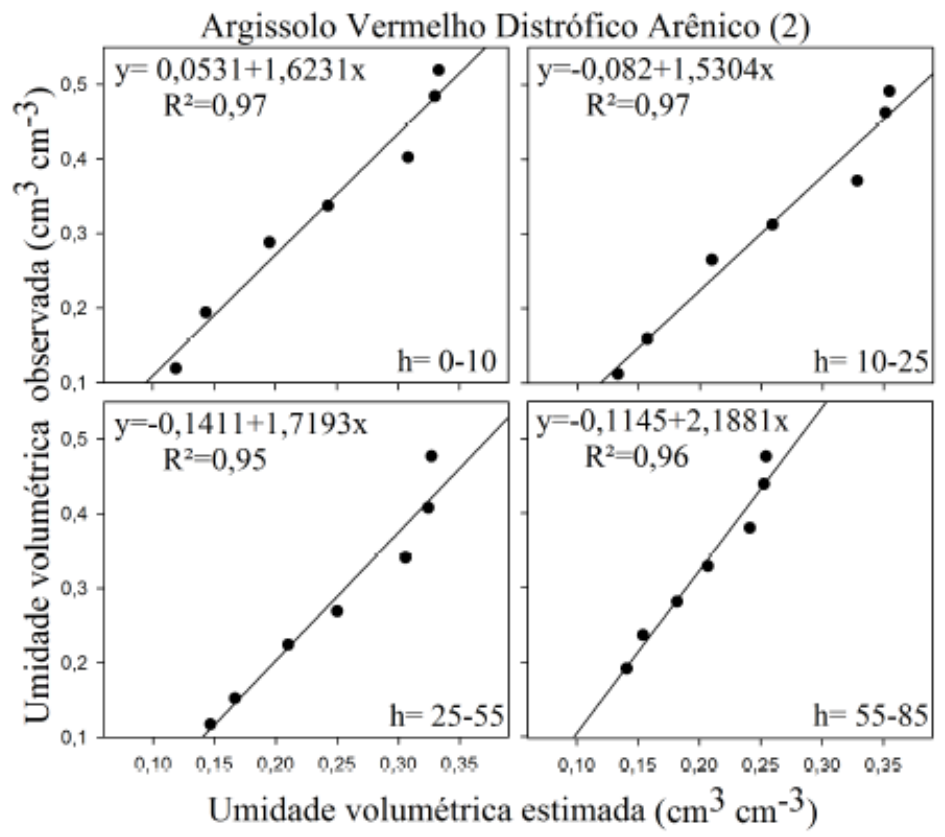

Figura 5. Representação gráfica da estimativa da umidade volumétrica obtida através do método direto, comparada com os valores de umidades obtidos através dos parâmetros gerados pelo Qualisolo para o Argissolo Vermelho distrófico arênico (2) nas diferentes profundidades. Graphical representation of the volumetric moisture estimation obtained by the direct method, compared to the values obtained by the parameters generated by the Qualisolo for the Typic Paleudult (2) at different depths

Latossolo Vermelho Distrófico Típico (3)

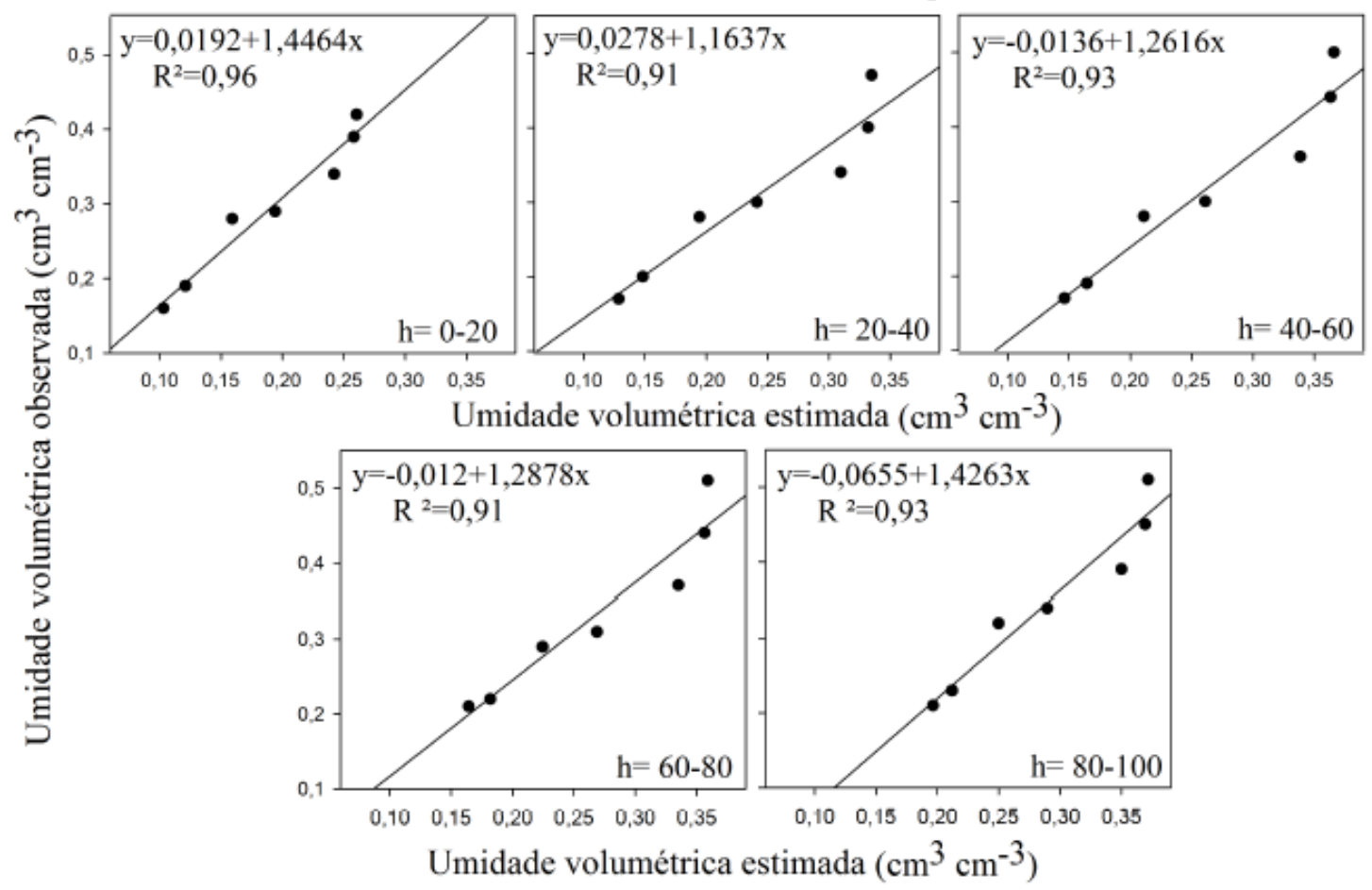

Figura 6. Representação gráfica da estimativa da umidade volumétrica obtida através do método direto, comparada com os valores de umidades obtidos através dos parâmetros gerados pelo Qualisolo para o Latossolo Vermelho distrófico típico (3) nas diferentes profundidades analisadas. Graphical representation of the volumetric moisture estimation 
obtained by the direct method, compared to the values obtained by the parameters generated by the Qualisolo for the Typic Hapludox (3) at different depths.

$\mathrm{Na}$ Tabela 3, estão apresentados os valores de R2, RQME, o EAM, índice d e índice c, para as diferentes profundidades analisadas em cada perfil de solo e também a análise da média do perfil.

Tabela 3. Valores do coeficiente de determinação $\left(\mathrm{R}^{2}\right)$, raiz do quadrado médio do erro (RQME), erro absoluto médio (EAM), índice de Willmott (d), índice de Camargo e Sentelhas (c) e desempenho, obtidos de duas classes de solo de três locais de coleta. Coefficient of determination values $\left(R^{2}\right)$, root mean square error (RMSE), mean absolute error (MAE), agree-ment index $(d)$, Camargo and Sentelhas index (c) and performance, obtained from two soil classes at three locations.

\begin{tabular}{|c|c|c|c|c|c|c|}
\hline Profundidade $(\mathrm{cm})$ & $\mathrm{R}^{2}$ & RQME & EAM & D & $\mathrm{c}$ & Desempenho \\
\hline \multicolumn{7}{|c|}{ Argissolo Vermelho distrófico arênico (1) } \\
\hline $0-5$ & 0,91 & 0,1 & 0,08 & 0,67 & 0,64 & Mediano \\
\hline $5-15$ & 0,90 & 0,09 & 0,08 & 0,71 & 0,68 & Bom \\
\hline $15-30$ & 0,87 & 0,09 & 0,08 & 0,7 & 0,66 & Bom \\
\hline $30-45$ & 0,94 & 0,1 & 0,09 & 0,7 & 0,68 & Bom \\
\hline $45-60$ & 0,95 & 0,12 & 0,11 & 0,64 & 0,62 & Mediano \\
\hline 60-75 & 0,94 & 0,11 & 0,09 & 0,68 & 0,66 & Bom \\
\hline Média do perfil & 0,94 & 0,1 & 0,09 & 0,95 & 0,92 & Ótimo \\
\hline \multicolumn{7}{|c|}{ Argissolo Vermelho distrófico arênico (2) } \\
\hline $0-10$ & 0,97 & 0,11 & 0,1 & 0,69 & 0,68 & Bom \\
\hline $10-25$ & 0,97 & 0,07 & 0,06 & 0,89 & 0,87 & Ótimo \\
\hline $25-55$ & 0,95 & 0,07 & 0,05 & 0,88 & 0,85 & Muito Bom \\
\hline $55-85$ & 0,96 & 0,14 & 0,13 & 0,61 & 0,6 & Sofrível \\
\hline Média do perfil & 0,97 & 0,1 & 0,08 & 0,91 & 0,89 & Ótimo \\
\hline \multicolumn{7}{|c|}{ Latossolo Vermelho distrófico típico (3) } \\
\hline $0-20$ & 0,96 & 0,11 & 0,1 & 0,6 & 0,59 & Sofrível \\
\hline $20-40$ & 0,91 & 0,07 & 0,07 & 0,84 & 0,8 & Muito Bom \\
\hline $40-60$ & 0,93 & 0,07 & 0,06 & 0,89 & 0,86 & Ótimo \\
\hline $60-80$ & 0,91 & 0,08 & 0,07 & 0,84 & 0,8 & Muito Bom \\
\hline $80-100$ & 0,93 & 0,07 & 0,06 & 0,84 & 0,81 & Muito Bom \\
\hline Média do perfil & 0,93 & 0,08 & 0,07 & 0,93 & 0,9 & Ótimo \\
\hline
\end{tabular}

Analisando em conjunto todos os solos estudados, foi possível verificar que a comparação entre os dados observados e estimados apresentaram resultados coerentes, sendo, que o índice c apresentou valores superiores a 0,59 onde foram classificados como sofrível. Para o solo Argissolo Vermelho distrófico arênico (2) (camada $10-25 \mathrm{~cm}$ ) e Latossolo Vermelho distrófico típico (3) (camada $40-60 \mathrm{~cm}$ ), o índice de $\mathrm{d}$ foi superior a 0,87 e 0,85, respectivamente, classificando o desempenho do modelo como ótimo (Tabela 3).

Para o Argissolo Vermelho distrófico arênico (1) pode-se verificar que o mesmo apresentou uma variação do desempenho de mediano a bom para as camadas avaliadas, conforme índice de desempenho (Tabela 3). A variação do desempenho foi de sofrível a 
ótimo para a análise entre as camadas para o Argissolo Vermelho distrófico arênico (2). Já para Latossolo Vermelho distrófico típico (3) o desempenho entre as camadas do solo variou de sofrível a ótimo, e através de uma análise geral da média do perfil dos solos estudados o desempenho encontrado foi ótimo. A estimativa de umidade apresentou os maiores valores do coeficiente de determinação e do índice de concordância de Willmott e os menores valores de EAM e RQME, mostrando uma boa concordância com os dados observados pelo método da câmara de Richards, com destaque para o Argissolo Vermelho distrófico arênico (2) de Santa Maria e o Latossolo Vermelho distrófico típico (3) de Santiago, pois apresentaram os melhores desempenhos.

Com base no trabalho realizado, sugere-se a aplicação deste método em estudos futuros que necessitam a obtenção da umidade do solo em potenciais acima de 0,1 MPa. Recomendase também avaliar a limitação do modelo quanto aos valores de umidade para baixas tensões, possibilitando a sua aplicação para a quantificação da macro e microporosidade, ponto de inflexão da curva de retenção de água no solo e consequentemente os cálculos dos índices de qualidade do solo, tais como o índice $\mathrm{S}$.

\section{CONCLUSÃO}

As umidades estimadas para a curva de retenção de água dos solos estudados nas tensões de 0,001, 0,006, 0,01, 0,033,0,1,0,5, 1,5 MPa, apresentaram valores inferiores as umidades determinadas pelo método da câmara de Richards, com variação de $10 \%$ para 1,5 MPa a 44\% para 0,001 MPa.

Para todos os solos o coeficiente de determinação foi superior a 0,87 na comparação com as umidades observadas.

Através da utilização da análise textural com dados de entrada para aplicação dos métodos para determinar a umidade volumétrica do perfil solo, em uma análise geral permitiu, foi possível encontrar resultados classificados com desempenho ótimo para os três solos.

A utilização do modelo Arya e Paris para gerar os parâmetros de Van Genuchten é uma boa alternativa para determinação da curva de retenção de água no solo.

\section{AGRADECIMENTOS}

O presente trabalho foi realizado com apoio da Coordenação de Aperfeiçoamento de Pessoal de Nível Superior - Brasil (CAPES) - Código de Financiamento 001.

\section{REFERÊNCIAS BIBLIOGRÁFICAS}

ANTINORO, C.; BAGARELlO, V.; FERRO, V.; GIORDANO, G.; IOVINO, M. A simplified approach to estimate water retention for Sicilian soils by the Arya-Paris model. Geoderma, Amsterdam, v. 213, n. 4, p.226-234, 2014. 
ARYA, L. M.; PARIS, J. F. A physicoempirical model to predict soil moisture characteristics from particle-size distribution and bulk density data. Soil Science Society of America Journal, Madison, v. 45, n. 6, p.1023-1030, 1981.

BARROS, A. H. C.; LIER, Q. D. J. V.; MAIA, A. D. H. N.; SCARPARE, F. V. Pedotransfer functions to estimate water retention parameters of soils in northeastern Brazil. Revista Brasileira de Ciência do Solo, Viçosa, v. 37, n. 2, p.379-391. 2013.

BARROS, V. R.; SOUZA, A. P.; FONSECA, D. C.; SILVA, L. B. D. Avaliação da evapotranspiração de referência na Região de Seropédica, Rio de Janeiro, utilizando lisímetro de pesagem e modelos matemáticos. Revista Brasileira de Ciências Agrárias, Recife, v. 4, n. 2, p.198-203, 2009.

CAMARGO, A. P.; SENTELHAS, P. C. Avaliação do desempenho de diferentes métodos de estimativa da evapotranspiração potencial no estado de São Paulo. Revista Brasileira de Agrometeorologia, Santa Maria, v. 5, n. 1, p.89-97, 1997.

CAMPELO JUNIOR, J. H.; DE AZEVEDO, E. C.; DE CARVALHO ALVES, M.; MELlO, D.; ALMEIDA LOBO, F.; AMORIM, R. S. S. Estimativa da retenção de água em Latossolos do Cerrado mato-grossense cultivados com algodão. Revista Agroambiente, on-line, v. 8, n. 3, p. 318-326, 2014. Disponível em: $<$ https://revista.ufrr.br/agroambiente/article/view/1902/1426>. Acesso em: 15 de agosto de 2017.

EMPRESA BRASILEIRA DE PESQUISA AGROPECUÁRIA - EMBRAPA. Sistema brasileiro de classificação de solos. Brasília: Embrapa informação Tecnológica, 2005. 374 p.

EMPRESA BRASILEIRA DE PESQUISA AGROPECUÁRIA - EMBRAPA. Sistema brasileiro de classificação de solos. Rio de janeiro: Embrapa-CNPS, 1999. 412 p.

GOMES, A. C. S. Estudo experimental e simulado da Cultura da soja em função de diferentes Níveis de irrigação. 2011. 166 f. Tese (Doutorado em Engenharia Agrícola) Universidade Federal de Santa Maria, Santa Maria, 2011.

KHODAVERDILOO, H.; HOMAEE, M.; VAN GENUCHTEN, M. T.; DASHTAKI, S. G. Deriving and validating pedotransfer functions for some calcareous soils. Journal of Hydrology, Amsterdã, v. 399, n. 1- 2, p.93-99, 2011.

LUCAS, J. F. R.; TAVARES, M. H. F.; CARDOSO, D. L.; CÁSSARO, F. A. M. Curva de retenção de água no solo pelo método do papel-filtro. Revista Brasileira de Ciência do Solo, Viçosa, v. 35, n.6, p. 1957-1973, 2011.

MELO, T. M.; BRETANHA, S. S. F.; PEREIRA, M.; CASTRO, N. M. R.; LOUZADA, J. A. S.; OLIVEIRA, M. H. C. Comparação de Modelos para Determinação Indireta da Curva de Retenção de Água no Solo: Aplicação em Solos da Bacia do Arroio Taboão, RS. Revista Brasileira de Recursos Hídricos, Porto Alegre, v. 20, n. 3, p. 571582, 2015.

NAIME, J. M.; VAZ, C. M. P.; MACEDO, Á. Automated soil particle analyzer based on gamma-ray attenuation. Computers and Electronics in Agriculture, Amsterdã, v. 31, n. 3, p.295-304, 2001. 
NASCIMENTO, P. S.; BASSOI, L. H.; PAZ, V. P. S.; VAZ, C. M. P; NAIME. J. M.; MANIERI, J. M. Estudo comparativo de métodos para a determinação da curva de retenção de água no solo. Revista Irriga, Botucatu, v. 15, n. 2, p.193-207, 2010.

NASCIMENTO, P. S. Análise do uso da curva de retenção de água no solo determinada por diferentes métodos em planilha de manejo de irrigação. 2009. 88 p. Dissertação (Mestrado em Ciências Agrárias), Universidade Federal do Recôncavo da Bahia, Cruz das Almas, Bahia, 2009.

NEBEL, A. L. C.; TIMM, L. C.; CORNELIS, W.; GABRIELS, D.; REICHARDT, K.; AQUINO, L. S.; PAULETTO, E. A.; REINERT, D. J. Pedotransfer functions related to spatial variability of water retention attributes for lowland soils. Revista Brasileira de Ciência do Solo, Viçosa, v. 34, n.3, p.669-680, 2010.

OLIVEIRA, Z. B. Perdas de água e variações na temperatura de um argissolo vermelho em função de diferentes quantidades de resíduos vegetais na superfície e espaçamentos entre linhas do feijoeiro. 2011. 121 f. Dissertação (Mestrado em Engenharia Agrícola) Universidade Federal de Santa Maria, Santa Maria, 2011.

REINERT, D. J.; REICHERDT, J. M. Coluna de areia para medir a retenção de água no solo - protótipos e teste. Revista Ciência Rural, Santa Maria, v. 36, n. 6, p.1931-1935, 2006.

REZAEE, L.; SHABANPOUR, M.; DAVATGAR, N. Estimating the soil water retention curve from soil particle size distribution using the Arya and Paris model for Iranian soils. Turkish Journal of Agriculture and Forestry, Ankara, v. 35, n. 6, p.649-657, 2011.

RICHARDS, L. A. Physical conditions of water in soil. In: BLACK, C. A.; EVANS, D. D.; WHITE, J. L.; ENSMINGER, L. E.; CLARK, F. E. Methods of soil analysis: physical and mineralogical properties, including statistics of measurements and sampling. Madison: American Society of Agronomy, Washington, 1965. cap. 8, p. 128-152.

SCORZA JÚNIOR, R. P.; SILVA, J. P.; RIGITANO, R. L. Simulation of moisture profiles in a Latossol in Dourados region, in the State of Mato Grosso do Sul, Brazil. Engenharia Agrícola, Jaboticabal, v. 30, n. 1, p.22-32, 2010.

SILVA, A. C.; ARMINDO, R. A. Importância das funções de pedotransferência no estudo das propriedades e funções hidráulicas dos solos do brasil. Multi-Science Journal, Urataí, v. 1, n. 5, p.31-37, 2016.

TORRES, R. R. Resposta à irrigação e à adubação nitrogenada em cobertura da cultura do girassol. 2014. 84 f. Dissertação (Mestrado em Engenharia Agrícola) - Universidade Federal de Santa Maria, Santa Maria, 2014.

VAN GENUCHTEN, M. T. H. A closed-form equation for predicting the hydraulic conductivity of unsaturated soils. American Soil Science Society, Madison, v. 44, n. 5, p.892-898, 1980.

VAZ, C. M. P.; NAIME, J. M.; MACEDO, A. Soil particle size fractions determined by gamma-ray attenuation. Soil Science, Baltimore, v. 164, n. 6, p.403-410, 1999. 
VAZ, C. M. P; NAIME, J. M.; SHINYA, V. T. Modelo de Arya e Paris para a estimativa de retenção de água com dados da granulometria dos solos. In: CONGRESSO BRASILEIRO DE CIÊNCIA DO SOLO, 31, 2007, Gramado. Anais... Porto Alegre: SBCS, 2007. 1 CDROM.

WILlMOTT, C. J.; ACKLESON, S. G.; DAVIS, R. E.; FEDDEMA, J. J.; KLINK, K. M.; LEGATES, D. R.; DONNELL, J.O.; ROWE, C. M. Statistics for the evaluation and comparision of models. Journal of Geophysical Research, Ottawa, v. 90, n. C5, p.89959005, 1985.

XIANG-WEI, H.; MING-NA, S.; HORTON, R. Estimating Van Genuchten model parameters of undisturbed soils using an integral method. Pedosphere, Nanjing, v. 20, n. 1, p.55-62, 2010. 